\title{
ABORDAGENS HÍBRIDAS E INCLUSIVAS E FORMAÇÃO DE PROFESSORES DE LÍNGUAS ADICIONAIS DO E PARA O SÉCULO XXI
}

\section{HYBRID AND INCLUSIVE APPROACHES IN ADDITIONAL LANGUAGE TEACHER EDUCATION IN AND FOR THE XXI CENTURY}

\author{
FINARDI, Kyria Rebeca \\ kyria.finardi@gmail.com \\ UFES - Universidade Federal do Espírito Santo
}

\begin{abstract}
RESUMO O estudo revisa tendências e abordagens contemporâneas no ensino de línguas adicionais (L2) ${ }^{1}$ tecendo considerações sobre a formação de professores do e para o século XXI. Para tanto, o estudo revisa abordagens inclusivas e híbridas que combinam o ensino tradicional presencial com o ensino a distância, com uso de tecnologias que podem promover o desenvolvimento da autonomia do educando e sua inclusão social por meio da mediação da língua e da tecnologia. O trabalho conclui com algumas considerações sobre a formação de professores de língua do e para o século XXI em relação ao uso de abordagens híbridas e inclusivas.

Palavras-chave: Abordagens híbridas. Abordagens inclusivas. Formação e educação de professores de línguas.
\end{abstract}

\begin{abstract}
ABSTRAC The study reviews trends and contemporary approaches in the teaching of additional languages (L2) discussing teacher education for and in the twenty-first century. With that aim, the study reviews inclusive and hybrid approaches that combine traditional classroom teaching approaches with distance learning, using technologies that can promote the development of students' autonomy and social inclusion through the mediation of language and technology. The paper concludes with some considerations on language teacher education in and for the twenty-first century in relation to the use of hybrid and inclusive approaches.
\end{abstract}

Keywords: Hybrid approaches. Inclusive approaches. Language teacher education.

\section{INTRODUÇÃO}

Vários autores como por exemplo Blommaert, (2010); Bourdieu; Thompson, (1991); Casotti; Finardi, (2016); Finardi, (2015); Finardi; Santos; Guimarães, (2016)

10 termo língua adicional é usado aqui para se referir a qualquer língua exceto a língua materna e a fim de evitar terminologias problemáticas como língua estrangeira/segunda língua. 
alertam para a íntima relação existente entre a globalização, o multilinguisimo e o ensino de línguas adicionais (L2). Parte dessa relação resulta do amplo acesso à informação promovido pela internet, (FINARDI; PORCINO, 2014; FINARDI; PREBIANCA; MOMM, 2013), pelas migrações sociais e econômicas (ORTIZ; FINARDI, 2015), bem como pela mobilidade acadêmica (ARCHANJO, 2016), esta última incentivada pela internacionalização do ensino superior (SHIN; TEICHLER, 2014).

$O$ ensino de L2 se relaciona à globalização e às tensões globais e locais resultantes dela de várias formas, sendo que uma delas é por meio da manutenção da coesão nacional promovendo o equilíbrio entre as línguas faladas em um determinado território como é o caso do ensino de línguas maternas, nacionais e estrangeiras na Suíça reportado em Finardi e Csillagh (2016). Outra interface do ensino de L2 em geral e do inglês em particular com a globalização reside na ampliação do acesso à informação disponível na internet (FINARDI; PREBIANCA; MOMM, 2013) e à educação online por meio dos cursos abertos e dirigidos a um público amplo (os MOOCs - Massive Online Open Courses) (FINARDI; TYLER, 2015).

Uma terceira interface do ensino de L2 com a globalização se refere à integração de migrantes e pessoas em situação de mobilidade por meio do ensino da L2 com a consequente inclusão social da diversidade linguística e cultural causada pela mobilidade acadêmica (ARCHANJO, 2016) e pelos fluxos migracionais (ORTIZ; FINARDI, 2015).

Não menos importante é a relação que o ensino de $\mathrm{L} 2$ em geral e do inglês em particular tem com a circulação da produção acadêmica nacional (FINARDI; FRANÇA, 2016), que por sua vez se relaciona intimamente ao processo de internacionalização do ensino superior (FINARDI; SANTOS; GUIMARÃES, 2016). Segundo Hamel (2013), há um viés tendencioso na produção acadêmica atual que beneficia países anglo-falantes. Essa afirmação é corroborada por autores como Vavrus e Pekol (2015) que alegam que a globalização/internacionalização do ensino superior afeta mais positivamente países do hemisfério norte do que países do eixo sul.

Nas fronteiras cada vez mais apagadas/questionadas do mundo globalizado, o ensino/uso de L2 e a formação de professores de L2 enfrentam vários desafios que se materializam em tensões globais/locais que vão desde a promoção de políticas linguísticas e educacionais, (FINARDI; ARCHANJO, 2015; FINARDI; PREBIANCA, 2014; 
GRIN, 2003; PINHEIRO; FINARDI, 2014; PORCINO; FINARDI, 2014; NICOLAIDES; TILIO, 2013) até o investimento na educação inicial e continuada de professores de línguas, (ALENCAR, 2016; FADINI, 2016; TILIO, 2014; FINARDI; DALVI, 2012; 2013). Tendo em vista os desafios apresentados por esse mundo globalizado e pela necessidade de integrar culturas e línguas para viver pacificamente nesse contexto, este artigo se propõe a refletir sobre o potencial de inclusão social de algumas abordagens de ensino de L2 e sobre a formação docente para o uso dessas abordagens no Brasil do século XXI.

\section{ABORDAGENS HÍBRIDAS E INCLUSIVAS}

Casotti e Finardi (2016) revisam duas abordagens de ensino de línguas que, segundo as autoras, podem ser inclusivas, quais sejam, a abordagem de ensino de conteúdos diversos por meio da língua (Content and Language Integrated Learning CLIL), (FINARDI; SILVEIRA; ALENCAR, 2016; ORTIZ; FINARDI, 2015) e a abordagem híbrida. A CLIL, segundo Coyle, Hood e Marsh (2010), é uma abordagem de ensino de conteúdos diversos por meio de uma língua veicular (que pode ser uma L2) e que segundo Alencar (2016) pode ser usada para ensinar L2 por meio de conteúdos diversos. Segundo Sharma (2010), a abordagem híbrida combina a instrução presencial com a aprendizagem online (GRAHAM, 2006; FADINI; FINARDI, 2015a; 2015b; FINARDI; PREBIANCA; SCHMITT, 2016; FINARDI, 2012; PREBIANCA; CARDOSO; FINARDI, 2014; PREBIANCA; VIEIRA; FINARDI, 2014; PREBIANCA; FINARDI; CARDOSO, 2015; SILVEIRA; FINARDI, 2015).

Segundo Finardi (2015), a CLIL pode ser usada em uma abordagem híbrida para ensinar uma L2 por meio de outro componente curricular ou conteúdo qualquer que não a língua em si, como ocorre no caso de usuários de MOOC ofertados em L2. Em um estudo sobre o papel das L2 nos MOOCs, Finardi e Tyler (2015) concluíram que a maioria deles (quase $90 \%$ ) é ofertado em inglês, tornando o acesso a esse tipo de educação limitado em razão do idioma no qual ele é ofertado. Como a interdisciplinaridade é parte integrante da proposta da abordagem CLIL (ALENCAR, 2016), Finardi (2015) sugere que ela pode ser usada no Brasil para ensinar L2 por meio de outros conteúdos, utilizando- 
se, para isso, de abordagens híbridas, inclusive com o uso/adaptação de MOOCs, num formato de sala de aula invertida, ou como complemento de aulas tradicionais.

No contexto mundial, principalmente na Europa e no Canadá, a CLIL já é usada como abordagem bilíngue para promover o multilinguismo e a inclusão social, como mostra o estudo de Ortiz e Finardi (2015) sobre o uso dessa abordagem, associada à tecnologia, para desenvolver o capital social e promover a inclusão social de imigrantes e refugiados na Suíça. Nesse estudo os autores mostram como a língua alvo (francês como L2) e a tecnologia (grupos de Facebook e Whatsapp), foram usados para integrar os refugiados/imigrantes em Genebra.

Tendo em vista a necessidade de incorporar tecnologias em metodologias de ensino, e vice-versa, Finardi, (2015) sugere o uso da abordagem CLIL com o auxílio de MOOCs planejados para o ensino de conteúdos diversos e adaptados para o ensino/aprendizado de L2 (FINARDI; SILVEIRA; LIMA; MENDES, 2016) num formato de sala de aula invertida (FADINI; FINARDI, 2015a, 2015b; FINARDI; PREBIANCA, SCHMITT, 2016) no que a autora chama de abordagem CLIL Invertida. A fim de discutir algumas possibilidades de uso dessas abordagens no ensino de línguas e na formação docente, este artigo passa agora a tecer algumas considerações sobre a formação de professores de L2 para o uso de abordagens híbridas e inclusivas como forma de enfrentar os desafios da educação do século XXI.

\section{FORMAÇÃO DE PROFESSORES DE L2 NO BRASIL DO SECULO XXI E ABORDAGENS HÍBRIDAS E INCLUSIVAS}

A LDB - Lei de Diretrizes e Bases da Educação Nacional - rege a educação e a formação docente em nível nacional no Brasil. Tendo em vista o tamanho do território brasileiro e a diversidade cultural e linguística que o compõe (FINARDI, 2016), Casotti e Finardi (2016) sugerem que a formação docente vise à inclusão e reflexão de como abordar essa formação.

Segundo Silva e Angelim (2006 apud CASOTTI; FINARDI, 2016), alguns professores ainda têm dificuldade em associar a teoria à prática do ensino de línguas uma vez que não compreendem muito bem como a diversidade linguística e cultural do 
Brasil precisa ser pensada no ensino de línguas. Casotti e Finardi (2016) sugerem que frequentemente observamos a exclusão no apagamento de traços que caracterizam as marcas dialetais decorrentes de diferenças sociais em sala de aula. No caso específico do ensino de L2, Finardi (2014) sugere que é necessário aceitar os falantes não nativos de L2, incluindo esses falantes no rol dos falantes de L2 "autorizados" a ditar normas e usos da língua.

Em relação à inclusão promovida pela incorporação de tecnologias na educação, Teixeira e Finardi (2013) alegam que a incorporação de tecnologias em práticas pedagógicas de formação de professores é necessária para preparar professores para enfrentar os desafios da educação do século XXI. Nesse sentido, Pereira e Dos Santos (2014) afirmam que um dos desafios da incorporação das tecnologias na educação é garantir que elas sejam associadas a práticas pedagógicas que efetivamente favoreçam o aprendizado. Paiva (2013) lembra ainda que a incorporação da tecnologia na educação deve ser feita por professores formadores, dando o exemplo e o modelo a ser seguido. No caso específico da incorporação de tecnologias no ensino/aprendizado de L2, Finardi e Porcino (2014) afirmam que a falta de incorporação de tecnologias em metodologias de ensino de L2 assim como a incorporação acrítica delas pode resultar em graves entraves para o aprendizado pleno da L2.

Sabemos das dificuldades de ensinar uma L2 na educação básica pública brasileira (TILIO, 2014) e que não se resumem à baixa carga horária destinada ao ensino de L2 e à formação de professores de L2 para a inclusão da diversidade. Em face desses desafios, por um lado, e da necessidade de contorná-los, por outro, a fim de garantir uma educação linguística de qualidade, penso que o uso de abordagens híbridas como a CLIL Invertida (FINARDI, 2015) possa ser uma solução relevante, ainda que parcial, para essa problemática.

Entretanto, e como nos mostra Alencar (2016) e Fadini (2016), a formação docente para o uso dessas abordagens ainda está muito aquém do desejado, seja pela falta de estrutura das instituições para a incorporação de tecnologias, seja pela falta de exemplo de professores formadores (PAIVA, 2013), seja pela falta de motivação e formação docente para a incorporação de tecnologias em práticas pedagógicas ou no caso 
específico da CLIL, pela falta de professores com proficiência suficiente tanto nos conteúdos diversos quanto na língua veicular, neste caso, a L2.

De fato, Alencar (2016) sugere que a falta de proficiência de professores de L2 em conteúdos diversos ou a falta de proficiência em conteúdos diversos de professores de L2 representa o maior obstáculo à implementação da abordagem CLIL no Brasil. No caso do uso da CLIL Invertida e tomando como base os resultados de Fadini (2016), podemos dizer que o maior obstáculo à implementação dessa abordagem reside na falta de formação de professores de L2 para a incorporação de tecnologias na educação e em suas práticas docentes.

Ainda que raros, temos alguns exemplos de tentativas de adotar abordagens inclusivas como a CLIL e as abordagens híbridas no ensino/aprendizado de L2 no contexto nacional. A seguir esses estudos serão revisados antes de tecer algumas considerações sobre a formação docente para seu uso.

Finardi, Silveira e Alencar (2016), por exemplo, elaboraram, implementaram e analisaram um plano de ensino que tinha como objetivo ensinar os conteúdos de "Primeiros Socorros" e " Ondas" por meio do inglês (L2) para estudantes da Escola de Marinheiros. O plano foi elaborado em parceria com os professores de Física e de Primeiros Socorros e implementado pelo professor de Inglês. Resultados desse estudo sugerem que a abordagem CLIL usada foi bem recebida/avaliada pelos alunos, mas que eles tiveram mais dificuldade com o plano elaborado para dar aula do conteúdo de "Ondas", que segundo os alunos era mais difícil uma vez que a maioria dos alunos tinha mais dificuldade na disciplina de Física, em comparação com outras disciplinas. Com base nesse resultado, Finardi, Silveira e Alencar (2016) sugerem que a elaboração de planos de ensino na abordagem CLIL deve ser feita de forma criteriosa levando em consideração a complexidade/dificuldade do conteúdo a ser ensinado por meio da L2.

Em relação ao uso de abordagens híbridas que combinam o ensino presencial com a aprendizagem online no Brasil, temos vários estudos que comprovam os benefícios dessa abordagem. Finardi (2012) revisou quatro estudos de caso da incorporação de tecnologias no ensino de L2: o caso do livro digital, do ensino mediado por computador (Computer Assisted Language Learning-CALL), de um aplicativo para gravação e reprodução de voz (Voicethread) e de um software para o ensino de inglês. 
Resultados desse estudo sugerem que a abordagem híbrida é uma combinação relevante do que há de melhor no ensino presencial e no ensino a distância.

Finardi, Prebianca, Schmitt e Andrade (2014) analisaram o potencial de um curso online de inglês (MEO) para o aprendizado de L2. Resultados desse estudo sugerem que apesar do curso oferecer oportunidades para o aprendizado de leitura e compreensão oral em L2, o mesmo não poderia garantir o aprendizado pleno de L2 que inclui o desenvolvimento de habilidades de produção oral e escrita, a menos que fosse usado no formato híbrido com a mediação do professor ou de outros falantes de L2.

Silveira e Finardi (2015) elaboraram, implementaram e analisaram um plano de ensino que tinha como objetivo desenvolver a autonomia e a habilidade de compreensão leitora em L2 dos alunos da Escola de Marinheiros por meio de tarefas online realizadas extra classe. Resultados desse estudo mostraram que a abordagem híbrida usada foi positiva no desenvolvimento da autonomia e da habilidade de compreensão leitora em L2.

Silveira e Finardi (2016) elaboraram, implementaram e analisaram um plano de ensino de L2 no formato de sala de aula invertida, também na Escola de Marinheiros. Resultados desse estudo mostraram que ainda que os alunos admitam as vantagens do aprendizado autônomo e online, eles acreditam que a presença/mediação do professor é essencial para o desenvolvimento pleno da habilidade de comunicação em L2.

Prebianca, Vieira e Finardi (2014) analisaram a percepção de alunos sobre o uso de um software para o ensino de L2 em relação às características pedagógicas e ergonômicas dele. Os resultados da percepção dos alunos sobre a experiência de aprendizado com esse curso online se assemelha à conclusão de Silveira e Finardi (2016) no que diz respeito às vantagens do uso de abordagens híbridas que combinam a mediação/presença do professor de L2. Outros estudos que chegaram à mesma conclusão são o de Prebianca, Cardoso e Finardi (2014), o de Prebianca, Vieira e Finardi (2014) e o de Prebianca, Finardi e Cardoso (2015) que analisaram as percepções de alunos submetidos à abordagem híbrida no ensino/aprendizado de L2.

Finardi, Prebianca e Schmitt (2016) analisaram o potencial de um curso online de inglês (MEO) para a abordagem híbrida conhecida como Sala de Aula Invertida. Resultados desse estudo corroboram resultados de Finardi, Prebianca, Schmitt e 
Andrade (2014) sobre o MEO e todos os outros estudos citados aqui sobre o uso de abordagens híbridas no que se refere à necessidade de mediação do professor de L2 ou de outros falantes de L2 para o desenvolvimento pleno das habilidades produtivas (fala e escrita) na língua alvo.

Os resultados desses estudos, tomados em conjunto, parecem apontar para a conclusão de que tanto a abordagem CLIL quanto outras abordagens híbridas oferecem um potencial relevante para o ensino/aprendizado de L2. Se, por um lado, aceitamos essa conclusão e, por outro lado, constatamos a falta de formação docente para o uso dessas abordagens (ALENCAR, 2016; FADINI, 2016), veremos que há uma lacuna a ser preenchida na formação de professores de L2 do e para o Brasil do século XXI. Segundo Arruda (2013), a solução não está em modificar o currículo dos cursos de licenciatura, mas sim em incluir discussões sobre essas abordagens nos cursos de formação docente.

\section{CONSIDERAÇÕES FINAIS}

Este estudo teve como objetivo refletir sobre abordagens inclusivas no ensino/aprendizado de L2 tecendo algumas considerações sobre a formação de professores de L2 no Brasil do e para o século XXI. Para tanto, o estudo revisou uma abordagem inclusiva de educação bilíngue, a CLIL e abordagens híbridas. O resultado da análise do uso dessas abordagens no contexto hodierno brasileiro permitiu chegar às seguintes conclusões: 1) a implementação da abordagem CLIL deve levar em consideração a complexidade/dificuldade do conteúdo a ser ensinado por meio da L2; 2) ainda em relação à implementação da abordagem CLIL, faz-se necessário pensar na formação de professores de L2 para que tenham proficiência em conteúdos diversos bem como na formação de professores de conteúdos diversos para que tenham proficiência na L2; 3) a implementação de abordagens híbridas é positiva para o desenvolvimento da autonomia do aluno, mas a fim de que possa desenvolver as habilidades de produção oral e escrita, é necessário haver a presença/mediação de um professor de L2 ou de outros falantes/aprendizes da língua alvo. Uma solução para isso seria usar as abordagens híbridas como complemento de aulas no ensino presencial tradicional como propõe, por exemplo, Finardi (2015) na abordagem CLIL Invertida. Finalmente, uma 
última conclusão que podemos tecer com base nos estudos revisados aqui é que a discussão sobre o uso dessas abordagens deve estar presente nos cursos de formação docente e, segundo Paiva (2013), também no exemplo de professores formadores.

\section{KYRIA REBECA FINARDI}

Mestre e doutora em Letras/Inglês, área de concentração Inglês e Linguística Aplicada, pela Universidade Federal de Santa Catarina (UFSC) com pós doutorado em Inglês pela Universidade de Genebra (Unige). Professora do Departamento de Linguagens, Cultura e Educação e dos Programas de Pós-Graduação em Linguística (PPGEL) e Educação (PPGE) da Universidade Federal do Espírito Santo (UFES). Membro do GT da Anpoll de Linguagem e Tecnologia e Coordenadora do NEPEHTE e programa Idiomas sem Fronteiras/UFES.

\section{REFERÊNCIAS}

ALENCAR, J G. Abordagem de ensino de língua inglesa por meio de conteúdos e formação de professores: apropriações, possibilidades e limitações. 2016. Dissertação de Mestrado, Programa de Pós Graduação em Educação, Universidade Federal do Espírito Santo, Vitória, 2016.

ARCHANJO, R. Moving globally to transform locally? Academic mobility and language policy in Brazil. Language Policy (Periodical Print), v. online, p. 1-22. 2016.

ARRUDA, E. P. Ensino e aprendizagem na sociedade do entretenimento: desafios para a formação docente. Educação, v. 36, n. 2, p. 232-239, 2013.

BLOMMAERT, J. The sociolinguistics of globalization. Cambridge University Press, Cambridge, 2010.

BOURDIEU, P.; THOMPSON, J. B. Language and symbolic power. Harvard University Press, Cambridge, 1991.

CASOTTI, J. ; FINARDI, K. Abordagens inclusivas no ensino de línguas: desafios para a formação docente. Revista (Con) textos Linguísticos, v. 10, n. 15, p. 63-76, 2016.

COYLE, D.; HOOD, P.; MARSH, D. CLIL - Content Language Integrated Learning. Cambridge: Cambridge University Press, 2010.

FADINI, K. Formação inicial de professores de inglês do e para o século XXI: os papéis da língua e da tecnologia. 2016. Dissertação (Mestrado) - Programa de Pós-Graduação em Linguística. Universidade Federal do Espírito Santo, Vitória, 2016. 
FADINI, K.; FINARDI, K. R. Affordances of Web 2.0 Interfaces for the Teaching/Learning of L2 in the Flipped Classroom. In: INTERNATIONAL CONFERENCE OF EDUCATION, RESEARCH AND INNOVATION, 2015, Sevilha. Anais.... Madri: IATED, 2015a, p. 10521058.

FADINI, K.; FINARDI, K. R. Web 2.0 Tools for the L2 Class and Beyond. In: END 2015 INTERNATIONAL CONFERENCE ON EDUCATION AND NEW DEVELOPMENTS PROCEEDINGS, 2015, Lisboa. Anais... Lisboa, World Institute for Advanced Research and Science (WIARS, Lisboa), 2015b, p.603-607.

FINARDI, K. R. Technology and L2 learning: hybridizing the curriculum. In: III CONGRESSO INTERNACIONAL ABRAPUI LANGUAGE AND LITERATURE IN THE AGE OF TEBHNOLOGY, 2012,Florianópolis. Anais... Florianópolis, Universidade Federal de Santa Catarina, 2012, p. 1-8.

FINARDI, K. R. The Slaughter of Kachru's Five Sacred Cows in Brazil: Affordances of the Use of English as an International Language. Studies in English Language Teaching, v. 2, n. 4, p. 401-411, 2014.

FINARDI, K. R. Language Policies and Internationalisation in Brazil: The Role(s) of English as an Additional Language. In: Lydia SCIRIHA. (Org.). International Perspectives on Bilingualism. 1ed. Newcastle upon Tyne: Cambridge Scholars Publishing, Cambridge, 2016, p. 79-90.

FINARDI, K. R.; PREBIANCA, G. V. V.; SCHMITT, J.; ANDRADE, D. F. Technology, English language teaching and internationalization at a crossroad: insights from the analysis of a virtual learning environment in Brazil. In: INTERNATIONAL CONFERENCE OF EDUCATION, RESEARCH AND INNOVATION, Anais... Sevilha: IATED, 2014, p. 112.

FINARDI, K. R.; DALVI, M. A. Encurtando as distâncias entre teoria-prática e universidade-escola no ensino de línguas e na formação de professores. Intersecções (Jundiaí), v. 5, n. 2, p. 115-127, 2012.

FINARDI, K. R.; DALVI, M. A. Crenças de professores de línguas sobre a formação inicial e continuada. Glauks (UFV), v. 13, n. 1, p. 113-134, 2013.

FINARDI, K. R.; PORCINO, M. C. Tecnologia e metodologia no ensino de inglês: impactos da globalização e da internacionalização. Desterros, v. 1, n. 66, p. 239-284, 2014.

FINARDI, K. R.; PREBIANCA, G. V. V.; MOMM, C. F. Tecnologia na Educação: o caso da internet e do Inglês como linguagens de inclusão. Cadernos do IL, n. 46, p. 193-208, 2013. 
FINARDI, K. R.; PREBIANCA, G. V. V. Políticas linguísticas, internacionalização, novas tecnologias e formação docente: um estudo de caso sobre o curso de Letras Inglês em uma universidade federal. Leitura (UFAL), v. 1, n.53, p. 129-154, 2014.

FINARDI, K. R.; SILVEIRA, N.; ALENCAR, J. G. First Aid and Waves in English as a Foreign Language: Insights from CLIL in Brazil. The Electronic Journal of Science Education, v. 20, n.3, p. 11-30, 2016.

FINARDI, K. R.; TYLER, J. The Role of English and Technology in the Internationalization of Education: Insights from the Analysis of Moocs. In: $7^{\text {th }}$ INTERNATIONAL CONFERENCE ON EDUCATION AND NEW LEARNING TECHNOLOGIES, 2015, Barcelona. Anais... Edulearn15 Proceedings. Barcelona: IATED, 2015, p. 11-18.

FINARDI, K. R. Current Trends in ELT and Affordances of the Inverted CLIL Approach. Studies in English Language Teaching, v. 3, n. 4, p. 326-338, 2015.

FINARDI, K. R.; ARCHANJO, R. Reflections of Internationalization of Education in Brazil. In: 2015 INTERNATIONAL BUSINESS AND EDUCATION CONFERENCE, 2015, Nova lorque. Anais... 2015 International Business and Education Conference Proceedings. Nova lorque: Clute Institute, 2015. p. 504-510.

FINARDI, K. R.; FRANÇA, C. O inglês na internacionalização da produção científica brasileira: evidências da subárea de linguagem e linguística. Intersecções (Jundiaí), v 10, n.15, p. 234-250, 2016.

FINARDI, K. R.; SANTOS, J. M.; GUIMARAES, F. A Relação entre Línguas Estrangeiras e o Processo de Internacionalização: Evidências da coordenação de letramento internacional de uma universidade federal. Interfaces Brasil/Canadá, v. 16, n.1, p. 233255, 2016.

FINARDI, K. R.; PREBIANCA, G. V.; SCHMITT, J. English distance learning: possibilities and limitations of MEO for the Flipped Classroom. Revista Brasileira de Linguística Aplicada, v. 16, n.2, p. 181-208, 2016.

FINARDI, K. R.; SILVEIRA, N.; LIMA, S.; MENDES, A. R. M. MOOC in the Inverted CLIL approach: hybridizing English teaching/learning. Studies in English Language Teaching, v. 4 , n. 4 , p. 473-493, 2016.

GRAHAM, C. Blended learning systems: definition, current trends, future directions. In: BONK, Curtis. J.; GRAHAM, Charles R. (Eds.). Handbook of Blended Learning: Global perspectives, local designs. San Francisco, CA: Pfeiffer Publishing, 2006. p. 41-54.

GRIN, F. Language planning and economics. Current issues in language planning, v. 4, n. 1, p. 1-66, 2003.

HAMEL, R. E. L'anglais, langue unique pour les sciences? Le rôle des modèles 
plurilingues dans la recherche, la communication scientifique et l'enseignement supérieur. Synergies Europe, v. 1, n. 8, p. 53-66, 2013.

NICOLAIDES, C. S.; TILIO, R. C. Políticas de ensino e aprendizagem de línguas adicionais no contexto brasileiro: o caminho trilhado pela ALAB. In: C. NICOLAIDES, K.A SILVA, R. TÍLIO, \& CH ROCHA (Orgs.), Política e Políticas Linguísticas, 2013. p. 285306.

ORTIZ, R.; FINARDI, K. Social inclusion and CLIL: evidence from la Roseraie. In: : INTERNATIONAL CONFERENCE OF EDUCATION, RESEARCH AND INNOVATION, 2015, Sevilha. Anais... Sevilha. ICERI2015 Proceedings. Madri: lated, 2015, p. 76607666 .

PAIVA, V. L. A formação do professor para uso da tecnologia. In: SILVA, K. A.; DANIEL, F. G.; KANEKO-MARQUES, S. M.; SALOMÃO, A. C. B. (Orgs). A formação de professores de línguas: novos olhares. Campinas, SP: Pontes Editores, 2013. p. 209-230

PEREIRA, R.; DOS SANTOS, M. Literatura, sociointeracionismo e gamificação: diálogos interdisciplinares a partir de objeto de aprendizagem digital. Sociopoética-Revista do Programa de Pós-Graduação em Literatura e Interculturalidade, v. 1, n. 13, p. 29-62, 2014.

PINHEIRO, L. M. S.; FINARDI, K. Políticas públicas de internacionalização e o papel do inglês: evidências dos programas CsF e IsF. In: II CONEL, 2014, Vitória. Anais.... Vitória: Programa de Pós Graduação em Linguística da Universidade Federal do Espírito Santo, 2014, p. 76-78.

PORCINO, M. C.; FINARDI, K. R. Globalization and internationalization in ELT: methodology, technology and language policy at a crossword in Brazil. In: INTERNATIONAL CONFERENCE OF EDUCATION, RESEARCH AND INNOVATION,2014, Madri. Anais.... Madri: IATED, 2014. p. 1-11.

PREBIANCA, G. V. V.; CARDOSO, G. L.; FINARDI, K. R. Hibridizando a educação e o ensino de inglês: questões de inclusão e qualidade. Revista do GEL, v. 11, n.2, p. 47-70, 2014.

PREBIANCA, G. V. V.; FINARDI, K. R.; CARDOSO, G. L. Ensino-aprendizagem em contextos híbridos: o que pensam os alunos sobre o uso da tecnologia em aulas de inglês no ensino médio integrado. Caminhos em Linguística Aplicada, v. 12, n.1, p. 95-119, 2015.

PREBIANCA, G. V. V.; VIEIRA, M.; FINARDI, K. R. Instrução gramatical na era da tecnologia: investigando diferentes abordagens para o ensino-aprendizagem de inglês no ensino médio integrado. Signum: Estudos da Linguagem, v. 17, n.1, p. 181-214, 2014.

SHARMA, P. Blended learning. ELT journal, v. 64, n. 4, p. 456-458, 2010. 
SHIN, J. C; TEICHLER, U. The future of the post-Massied university at the crossroads. Heidelberg: Springer International Publishing, 2014.

SILVEIRA, N.; FINARDI, K. R. Hybridizing L2 learning: insights from an intact class experience. In: END 2015 - INTERNATIONAL CONFERENCE ON EDUCATION AND NEW DEVELOPMENTS, 2015, Porto. Anais.... Lisboa: World Institute for Advanced Research and Science (WIARS), 2015. p. 593-597.

SILVEIRA, N.; FINARDI, K.R. Hybridizing L2 learning: affordances of the inverted class approach with online tasks. In: CARMO, Mafalda. (Org.). Education Applications \& Developments II Advances in Education and Educational Trends Series. 1ed. Porto: In Science Press, 2016, v. 2, p. 91-100.

TEIXEIRA, D.; FINARDI, K. R. TICs no ensino presencial: evidências de um curso de formação continuada na Universidade Federal do Espírito Santo. Contextos Linguísticos, v. 7, n. 8.1 , p. 79-96, 2013.

TILIO, R. C. Língua estrangeira moderna na escola pública: possibilidades e desafios. Educação \& Realidade, v. 39, n. 3, p. 925-944, 2014.

VAVRUS, F.; PEKOL, A. Critical Internationalization: moving from theory to practice, FIRE: Forum for International Research in Education, v. 2, n. 2, p. 5-21, 2015. 